\title{
Audio fade-out profile shaping for interactive multimedia
}

\author{
Lucian Lupşa-Tătaru \\ Faculty of Electrical Engineering and Computer Science, \\ "Transilvania" University of Braşov, Bd. Eroilor No. 29, Braşov, Romania, \\ lupsa@programmer.net, lucian.lupsa@unitbv.ro
}

\begin{abstract}
The present investigation refers an effective technique of shaping the audio fade-out profile in the current context of high demand for interactive multimedia. A rational function is employed in order to bring forth a highly versatile fade-out transition. To emphasize that the audio volume, i.e. the output of the selected rational function, is returned without noticeable delays during the fading process, a straightforward implementation in pure JavaScript is put forward in the paper, taking into account the most unfavorable case from the computational point of view.
\end{abstract}

Keywords. Digital multimedia, interactive multimedia, audio effects, audio fade, fade profile, HTML5, JavaScript.

\section{Introduction}

The increasing demand for interactive multimedia, as the main form of communicating information such as audio and video content, respectively, calls for switching from high-performance computing to reactive computing. Hence, effective algorithms have to be developed and implemented to ensure quick responses, without notable delays, so that the user can have control of the multimedia content. [1]-[6].

Streaming media content on the Internet implicitly asks for applying special effects, in real-time, in response to various events. The major challenge comes in relation to streamed audio and video content, when the well-known techniques suitable for off-line processing need to be replaced with more effective ones in order to achieve the goal of software efficiency and, eventually, heighten the level of interactivity [2]-[8]. When audio content is delivered to the user, the interactivity is usually managed via the standard audio controls, i.e., the "play", "pause", "seeking" and "volume" controls [5], [6]. Advanced interactivity with audio objects can be attained by generating sound effects in response to user intervention [2]-[4].

The widely used sound effects are represented by the audio fades (fade-in, fade-out, fade-up, fadedown), which are applied in order to smooth the user listening experience and avoid hearing damage by exposing the user to events encompassing sudden changes in the audio level. However, when streaming digital online audio/video content of long duration, the user often interacts by alternatively accessing the "play" and "pause" media controls [5], [6]. In this situation, to heighten the multimedia experience, a real-time implementation that performs the initiation of a fade-in audio effect whenever the playback starts is provided in [9]. In the same context of incorporating strong interaction with the user, the present investigation advances an effective procedure of shaping the audio fade-out profile with a view to reactive computing. The intention here is to provide a highly customizable fade-out 
transition as an alternative to the sudden transition to silence, received by plainly accessing the "pause" standard media control.

The techniques of customizing the fade-out curves in the off-line mode are well-established, being mainly based on employing transcendental functions (logarithm, exponential, sine) to impose the timerelated evolution of the audio volume. More precisely, the very common fade-out profiles, received in various audio editors, are of logarithmic, exponential and S-curve type, with the S-curve shape being preferred for providing a more musical sounding [10]-[14]. In Fig. 1, Fig. 2 and Fig. 3 are illustrated the most frequently used fade-out shapes, received in Audacity audio editor with the amplitude scale being the default linear scale (a level of 0 designates silence) [10], [11].

Notwithstanding, real-time audio volume processing can fail to complete if various transcendental functions are employed to shape the fade curve. Hence, to ensure that the audio volume is computed and returned without notable delays in the course of fading-out, we employ here a rational function, i.e. a function provided by a rational fraction, to enforce the time-related evolution of the audio volume during the fading process. A plain JavaScript implementation is advanced in order to highlight the suitability of the adopted shaping technique with reactive computing.

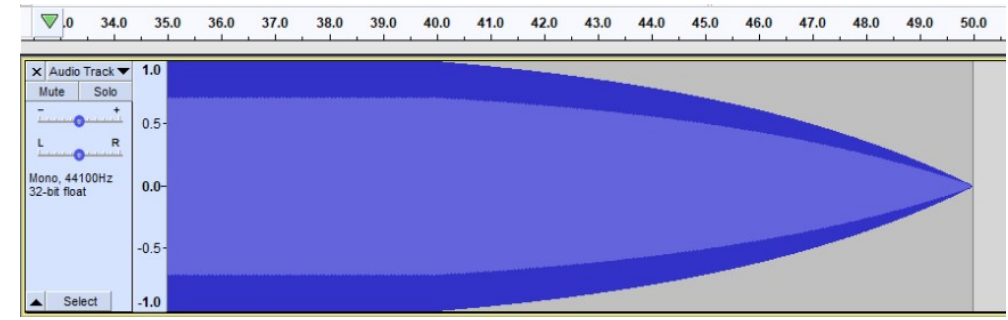

Fig. 1. A $10 \mathrm{~s}$ fade-out effect of logarithmic shape, received in Audacity audio editor by adopting the default linear scale.

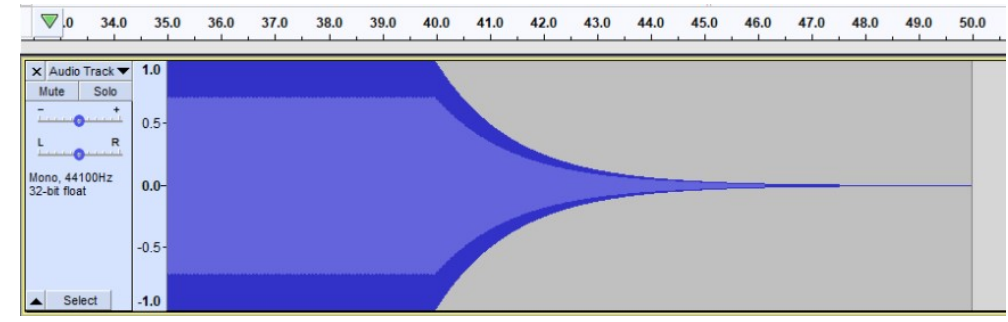

Fig. 2. A $10 \mathrm{~s}$ fade-out effect of exponential shape, received in Audacity audio editor by adopting the default linear scale.

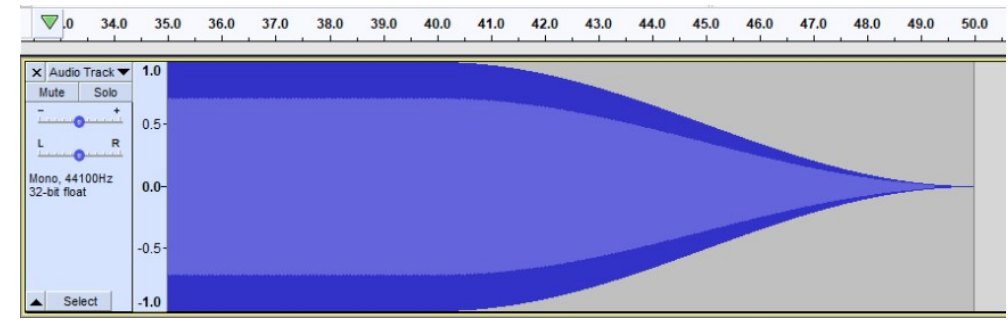

Fig. 3. A 10 s fade-out effect of S-curve shape, received in Audacity audio editor by adopting the default linear scale. 


\section{Shaping the fade-out profile}

As aforementioned, the widely used audio fade-out profiles are of logarithmic, exponential and Scurve type. Thus, the regular fade-out profiles are conventionally customized individually by means of transcendental functions, i.e., via the logarithm, exponential and sine function, respectively.

To minimize the delay between receiving the input, which is the difference of the current time within audio content and the instant of fade initiation, and returning the output, that is the appropriate audio volume, we consider here that the fade-out profile is yielded by the following rational function:

$$
\left\{\begin{array}{l}
v(\tau)=\frac{\tau^{k}-\alpha}{\beta \tau^{k}-\gamma} \\
\tau \in\left[0, \tau_{f}\right], \quad k \in\{1,2,3,4\}
\end{array}\right.
$$

wherein:

$v$ - denotes the audio volume (output),

$\tau$ - the difference of the playback time and the instant of fade-out initiation, i.e. the input

$$
\tau=t-t_{\text {ref }}
$$

$\tau_{f}$ - designates the fade-out length,

$\alpha, \beta, \gamma$ - coefficients that will be computed by taking into account the initial volume, the volume imposed at the fade midpoint, and the fact that the value of 0 indicates silence.

One observes that for $k=1$, the rational fraction (1) comes to be identical to the one given in [15]. Nevertheless, it will be shown that for $k \neq 1$ that is $k \in\{2,3,4\}$, function (1) enables the user to have more control in customizing the fade-out shape by incorporating attributes of the S-curve profile, hence allowing for a more musical sounding [10]-[12].

Denoting the initial volume, i.e., the audio volume occurring at the fade-out initiation by means of variable $v_{0}$, then the following conditions have to be met:

$$
\left\{\begin{array}{l}
v(0)=v_{0} \\
v\left(\tau_{f}\right)=0
\end{array}\right.
$$

Considering now (1) and (3), one eventually obtains the coefficient $\alpha$ in terms of fade length and the coefficient $\gamma$ in terms of fade length and initial volume, respectively:

$$
\begin{aligned}
& v(0)=v_{0} \Leftrightarrow \alpha / \gamma=v_{0}, \\
& v\left(\tau_{f}\right)=0 \Leftrightarrow\left\{\begin{array}{l}
\alpha=\tau_{f}^{k}, \\
\beta \tau_{f}^{k}-\gamma \equiv \alpha \beta-\gamma \neq 0
\end{array}\right. \\
& \gamma=\alpha / v_{0}=\tau_{f}^{k} / v_{0} .
\end{aligned}
$$

Accordingly, with $\alpha=\tau_{f}^{k}$ and $\gamma=\tau_{f}^{k} / v_{0}$, function (1) becomes:

$$
v(\tau)=\frac{\tau^{k}-\tau_{f}^{k}}{\beta \tau^{k}-\frac{\tau_{f}^{k}}{v_{0}}}=v_{0} \frac{\tau^{k}-\tau_{f}^{k}}{\beta v_{0} \tau^{k}-\tau_{f}^{k}} .
$$


To express now the coefficient $\beta$ in (5), we impose:

$$
\begin{gathered}
v\left(\tau_{h}\right)=v_{h}=\rho_{h} v_{0}, \\
\rho_{h}=v_{h} / v_{0} ; \quad 0<\rho_{h}<1
\end{gathered}
$$

wherein $\tau_{h}=\tau_{f} / 2$ is the fade-out midpoint and, thus, $\rho_{h}$ is the ratio between the audio volume at the midpoint and the initial audio volume, detected at the beginning of fading-out. Hence,

$$
v\left(\tau_{f} / 2\right)=\rho_{h} v_{0} .
$$

Taking now into account relations (5) and (7), we successively obtain:

$$
\begin{gathered}
\frac{\left(\tau_{f} / 2\right)^{k}-\tau_{f}^{k}}{\beta v_{0}\left(\tau_{f} / 2\right)^{k}-\tau_{f}^{k}}=\rho_{h}, \\
\frac{2^{-k}-1}{2^{-k} \beta v_{0}-1}=\rho_{h}, \\
\frac{2^{k}-1}{2^{k}-\beta v_{0}}=\rho_{h}, \\
\beta=\frac{1-2^{k}\left(1-\rho_{h}\right)}{\rho_{h} v_{0}} .
\end{gathered}
$$

With the audio volume as the output of (1), the instantaneous rate of change of the audio volume is given by

$$
\frac{d v}{d \tau}=\frac{k(\alpha \beta-\gamma) \tau^{k-1}}{\left(\beta \tau^{k}-\gamma\right)^{2}}
$$

In order to depict the profile of a fade-out effect, function (1) has to be strictly decreasing. Having in view (12), we obtain the equivalence relation:

$$
\frac{d v}{d \tau}<0 \Leftrightarrow \alpha \beta-\gamma<0 .
$$

The three coefficients in (13) are yielded by relations (4) and (11), Thus, one gets:

$$
\begin{aligned}
\alpha \beta-\gamma & =\tau_{f}^{k} \frac{1-2^{k}\left(1-\rho_{h}\right)}{\rho_{h} v_{0}}-\frac{\tau_{f}^{k}}{v_{0}} \\
& =\frac{\tau_{f}^{k}}{v_{0}}\left(2^{k}-1\right)\left(1-\frac{1}{\rho_{h}}\right) .
\end{aligned}
$$

Since $k \geq 1$ and $0<\rho_{h}<1$, it follows that quantity (14) is negative, which proves that the employment of rational function (1) to shape the fade profile forces the decaying with time of the audio volume. 


\section{The fade-out curves}

Similar to the exemplifications of Fig. 1, Fig. 2 and Fig. 3, respectively, we consider the initial audio volume of value $v_{0}=1$ and the fade-out length of value $\tau_{f}=10 \mathrm{~s}$. Apart from these settings, the fade-out shape remains highly customizable since both parameter $k$ in (1) and the ratio $\rho_{h}$ in (6) can be altered. However, having in view that, in the present case, the initial volume has the value of 1 , it follows that quantity $\rho_{h}$, defined as the ratio between audio volume at the midpoint and the initial audio volume, comes to be identical to the volume at the fade midpoint, i.e.,

$$
\rho_{h} \equiv v_{h} \equiv v\left(\tau_{f} / 2\right) .
$$

Fig. 4 up to Fig. 7 illustrate the fade-out curves for the adopted circumstance. Each of the four data charts emphasizes, for a certain value of $k$, the profiles corresponding to $\rho_{h} \in\{0.05,0.5,0.8\}$.

Fig. 4 indicates that for the simplified case of $k=1$ in (1), besides the linear fade, corresponding to $\rho_{h}=0.5$, the resulted fade-out effects can act either similar to the exponential fade-out $\left(\rho_{h}=0.05\right)$ or as the fade-out effect of logarithmic profile $\left(\rho_{h}=0.8\right)$ [15].

The fade curves in Fig. 5, Fig. 6 and Fig. 7, respectively, have been obtained by successively increasing the value of $k$ in (1). One observes that the fade-out profiles shown in Fig. 5 up to Fig. 7, corresponding to $\rho_{h}=0.5$, now incorporate attributes of S-curve shape: flattened starting, flattened ending [10]-[12]. Also, the S-curve attributes are present in the profiles of Fig. 6 and Fig. 7, which correspond to $\rho_{h}=0.05$.

Thus, one may conclude that, both in the beginning and in the ending region of the effect, the absolute value of the rate of change of audio volume decreases with parameter $k$ in (1).

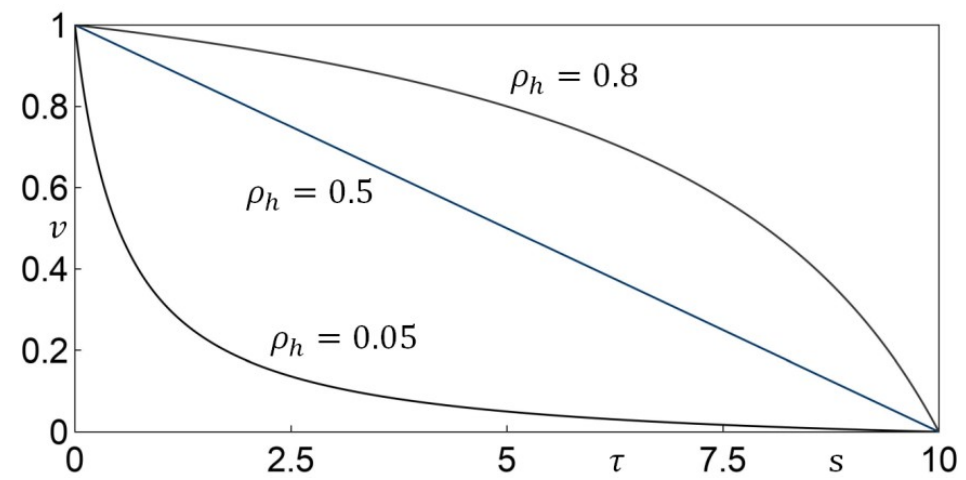

Fig. 4. Fade-out curves for $k=1$ in (1) and $\rho_{h} \equiv v_{h} \in\{0.05,0.5,0.8\}$

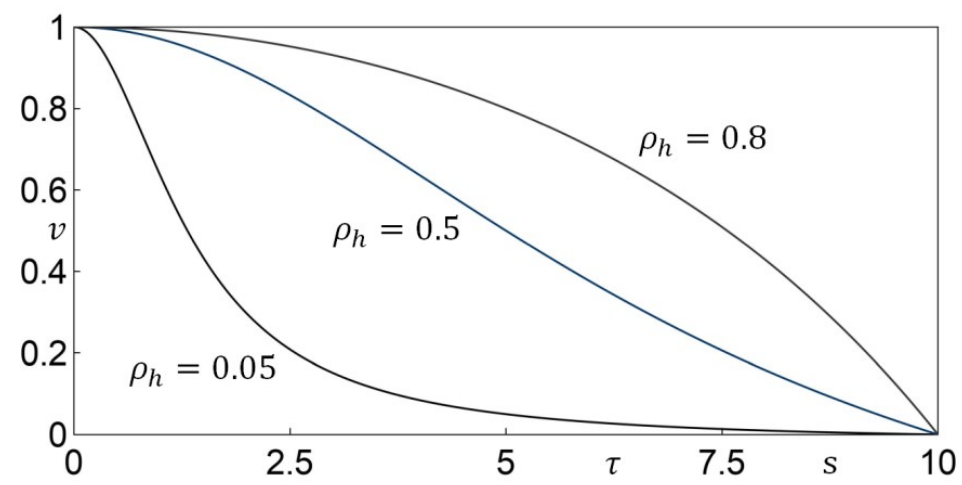

Fig. 5. Fade-out curves for $k=2$ in (1) and $\rho_{h} \equiv v_{h} \in\{0.05,0.5,0.8\}$. 


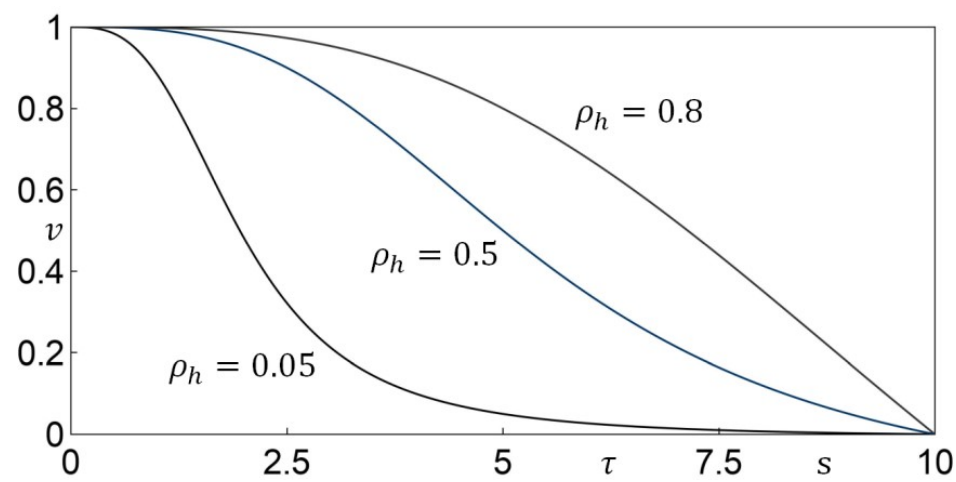

Fig. 6. Fade-out curves for $k=3$ in (1) and $\rho_{h} \equiv v_{h} \in\{0.05,0.5,0.8\}$.

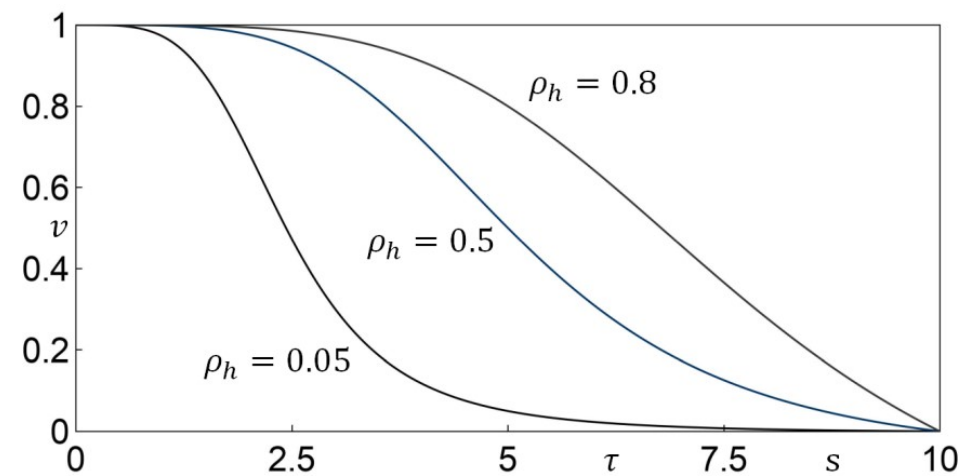

Fig. 7. Fade-out curves for $k=4$ in (1) and $\rho_{h} \equiv v_{h} \in\{0.05,0.5,0.8\}$

With the instantaneous rate of change of audio volume given by (12), wherein the coefficients are yielded by (4) and (11), one obtains the following relations corresponding to fade initiation:

$$
\begin{gathered}
\left.\frac{d v}{d \tau}(0) \equiv \frac{d v}{d \tau}\right|_{\tau=0}=\frac{v_{0}}{\tau_{f}}\left(1-\frac{1}{\rho_{h}}\right)<0 \\
k=1
\end{gathered}
$$

and, also, the rate of change at the fade-out ending:

$$
\begin{gathered}
\left.\frac{d v}{d \tau}\left(\tau_{f}\right) \equiv \frac{d v}{d \tau}\right|_{\tau=\tau_{f}}=\frac{k(\alpha \beta-\gamma) \tau_{f}^{k-1}}{\left(\beta \tau_{f}^{k}-\gamma\right)^{2}}, \\
\frac{d v}{d \tau}\left(\tau_{f}\right)=\frac{k(\alpha \beta-\gamma) \tau_{f}^{k-1}}{(\alpha \beta-\gamma)^{2}}, \\
\frac{d v}{d \tau}\left(\tau_{f}\right)=\frac{k \tau_{f}^{k-1}}{\frac{\tau_{f}^{k}}{v_{0}}\left(2^{k}-1\right)\left(1-\frac{1}{\rho_{h}}\right)},
\end{gathered}
$$




$$
\frac{d v}{d \tau}\left(\tau_{f}\right)=-\left|\frac{d v}{d \tau}\left(\tau_{f}\right)\right|, \quad\left|\frac{d v}{d \tau}\left(\tau_{f}\right)\right|=\frac{k}{2^{k}-1} \frac{v_{0}}{\left(\rho_{h}^{-1}-1\right) \tau_{f}}
$$

As noticed, similar to the case of Fig. 1, Fig. 2 and Fig. 3, the fade-out curves illustrated in Fig. 4 up to Fig. 7 have been obtained by adopting the linear scale, with the audio volume of 0 indicating silence. This is because the default linear scale is suitable not only for performing audio editing but also for software implementation [10], [11].

To emphasize the psychological response in relation to sound, the audio fade-out curves depicted in Fig. 8 to Fig. 11 have been received by switching from the audio volume as the data chart ordinate to the audio level in dB FS (decibel relative to full scale) that is [16]-[18]:

$$
L_{v}=20 \log _{10} v
$$

wherein audio volume $v$ is yielded by (1). The selected ordinates interval in Fig. 8 up to Fig. 11 ranges from $-48 \mathrm{~dB}$ to $0 \mathrm{~dB}$, where the audio level of $0 \mathrm{~dB}$ corresponds to volume value of 1 i.e. the adopted maximum value of Fig. 4 to Fig. 7 , whilst the level of $-48 \mathrm{~dB}$ is associated here with an audio volume of about 0.004 . Since both in the set of curves within Fig. 4 to Fig. 7 and the set of curves within Fig. 8 to Fig. 11, quantity $\rho_{h}$ is identical to the audio volume at the fade midpoint, it follows that the audio levels in Fig. 8 to Fig. 11, associated with $\rho_{h} \equiv v_{h} \in\{0.05,0.5,0.8\}$, are given by

$$
L_{h}=20 \log _{10} v_{h} ; \quad v_{h} \in\{0.05,0.5,0.8\}
$$

that is, approximately, $L_{h} \in\{-26 \mathrm{~dB},-6 \mathrm{~dB},-2 \mathrm{~dB}\}$.

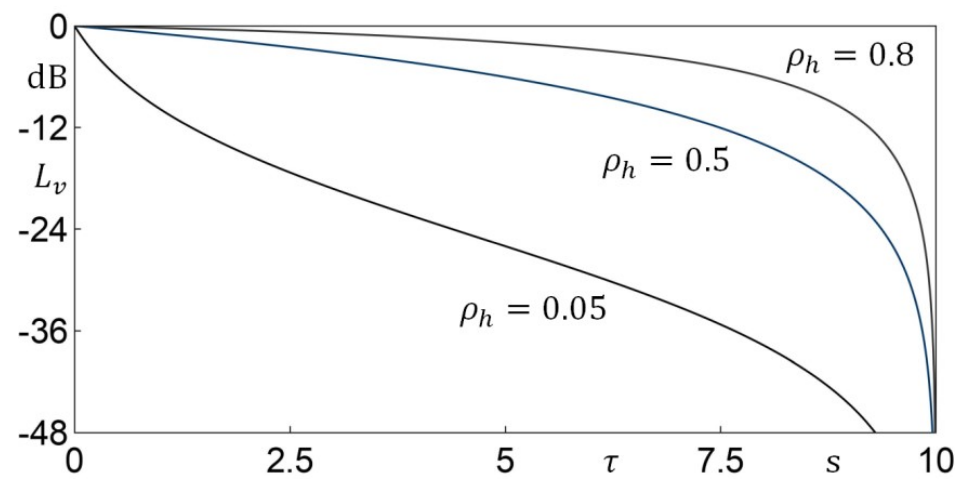

Fig. 8. Fade-out curves for $k=1$ in (1) and audio level (21) within interval [ $-48 \mathrm{~dB}, 0 \mathrm{~dB}$ ].

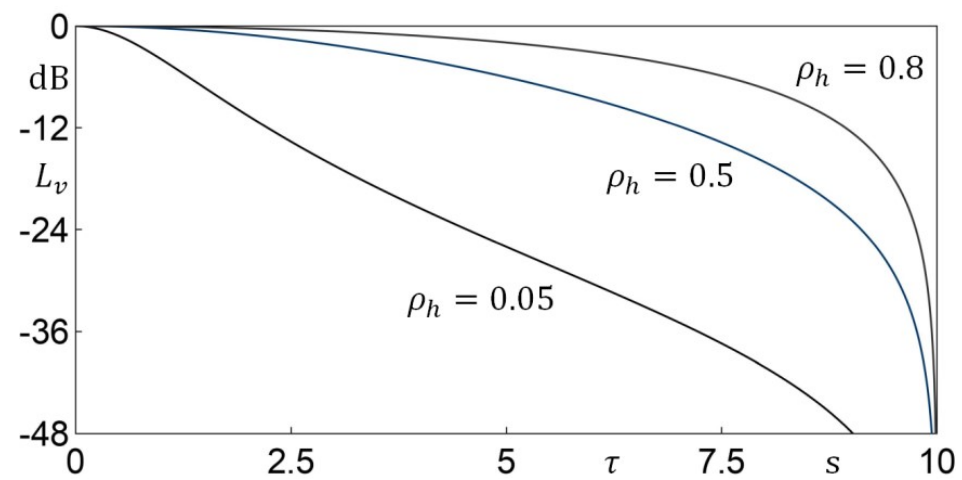

Fig. 9. Fade-out curves for $k=2$ in (1) and audio level (21) within interval [-48 dB, $0 \mathrm{~dB}$ ]. 


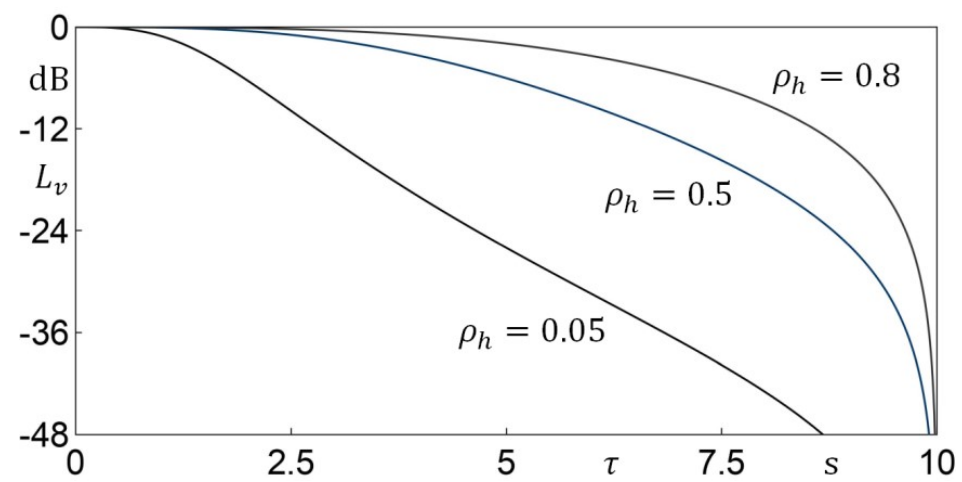

Fig. 10. Fade-out curves for $k=3$ in (1) and audio level (21) within interval [-48 dB, $0 \mathrm{~dB}]$.

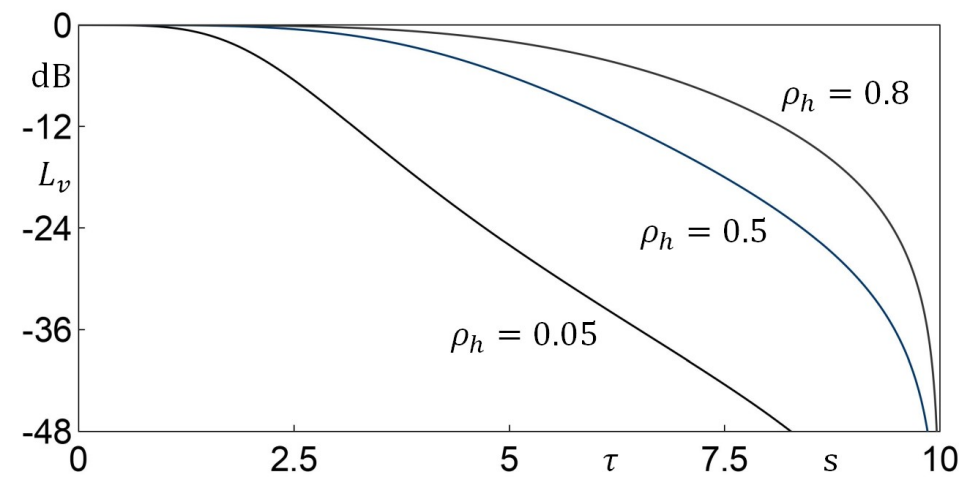

Fig. 11. Fade-out curves for $k=4$ in (1) and audio level (21) within interval [-48 dB, $0 \mathrm{~dB}]$.

\section{Shaping technique validation}

To prove the effectiveness of the proposed technique of audio fade customization in the context of interactive computing, we put forward an HTML5/JavaScript application, wherein the discretization is accomplished via the "setInterval()" method contained by the "window" object.

With the "src" property of the HTML DOM "audio" object pointing to the location of the audio sample, yielded by the user, the code here can be tested in any major browser. It has to be emphasized that, from the computational point of view, the application advanced in the paper corresponds to the most unfavorable situation, for which we have $k=4$ in the rational fraction (1).

Interactivity is managed by means of two "button" objects (elements), as follows:

- initially, the second button, i.e. the one that allows the fading-out initiation, is disabled (made unclickable);

- if the user clicks on the first button, i.e. the one that allows playback at the maximum (default) volume, then the audio content starts playing, and the first button is disabled whilst the second one is made clickable;

- if the user clicks on the second button then the fading-out effect is initiated and the second button is disabled;

- at the end of fading process, the audio content is paused and the first button, which allows regular playback, is made clickable;

- if there is not enough room for performing fading-out then both buttons are disabled.

The code of the application used for checking the validity of the suggested technique of fade-out profile shaping is given next. 


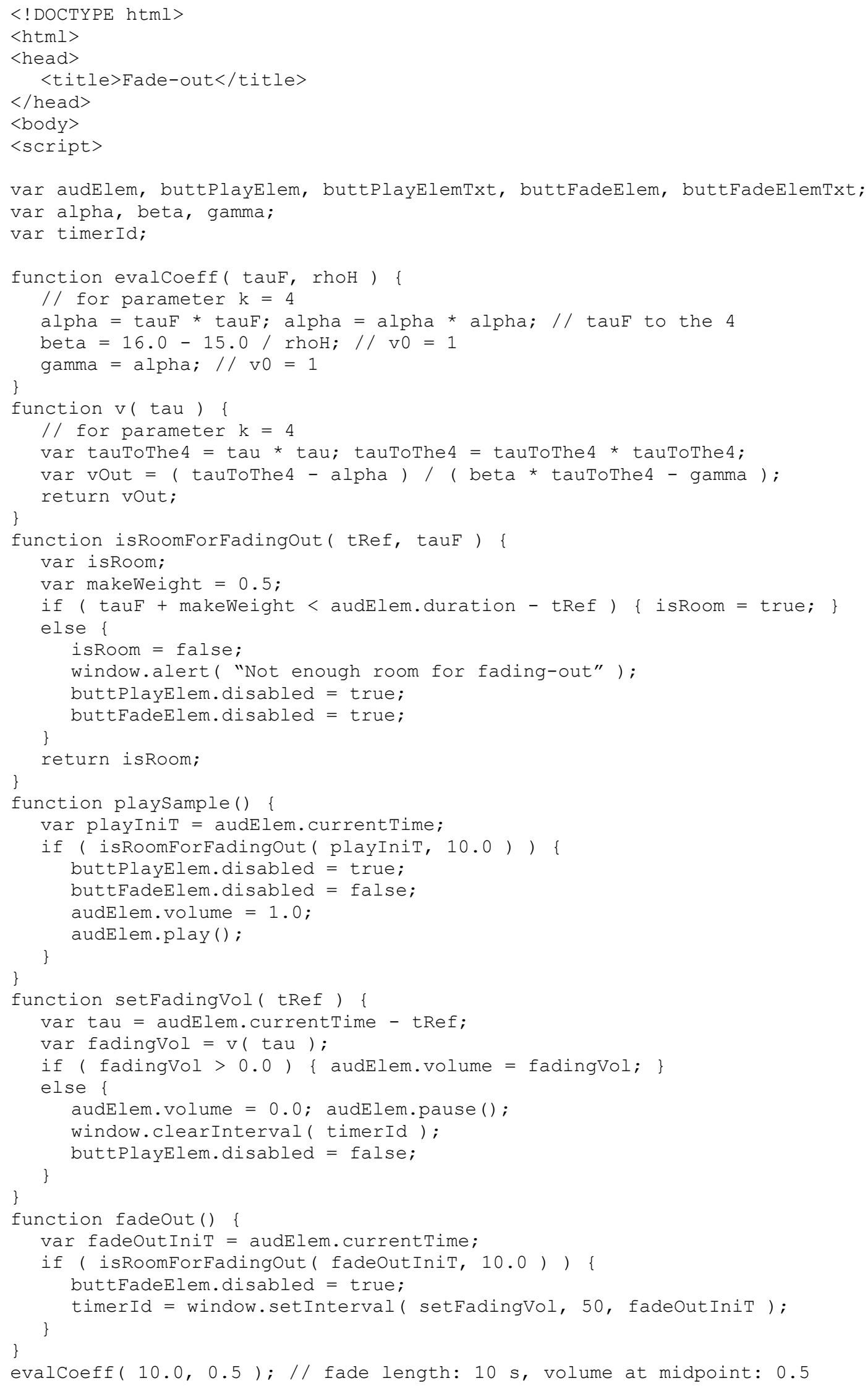




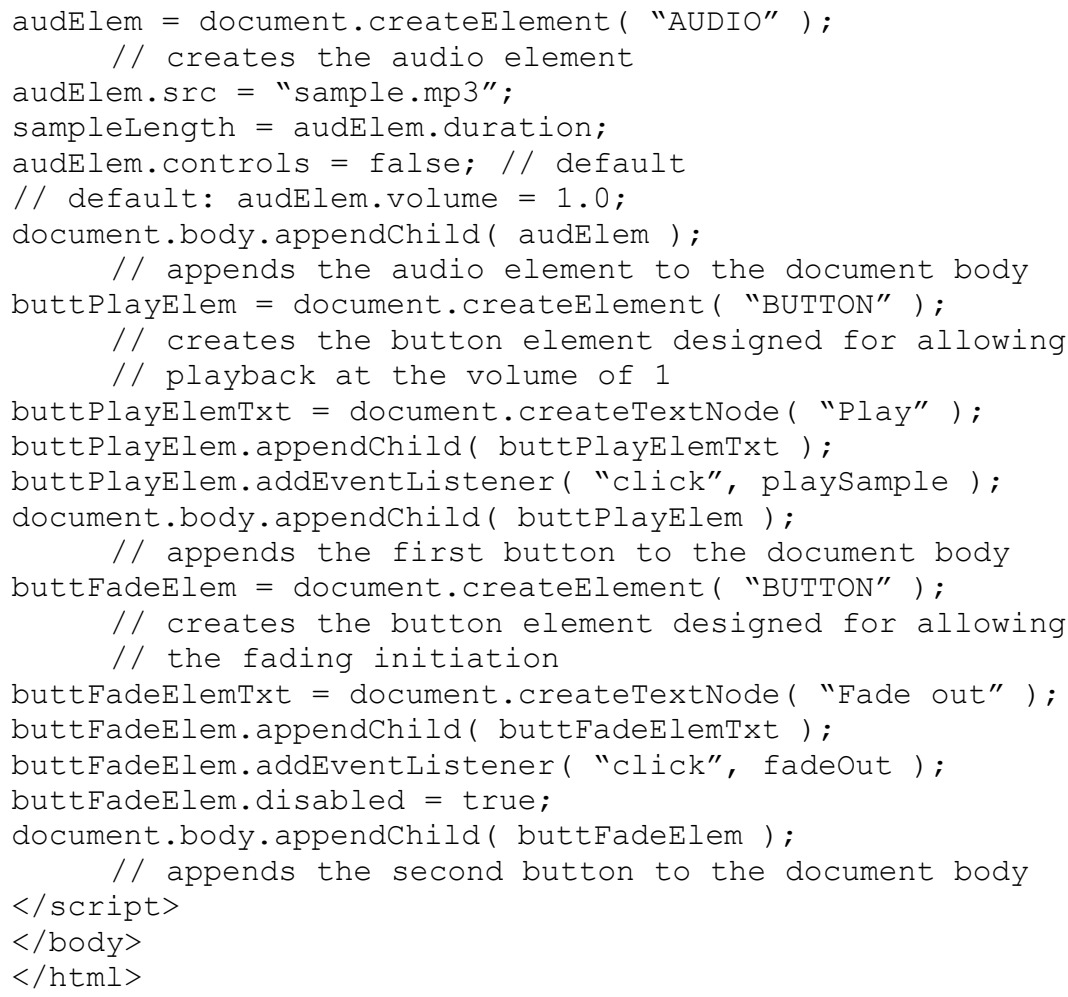

The code clearly indicates that the three coefficients encompassed by rational fraction (1) are designated by the global variables "alpha", "beta" and "gamma", and are computed by calling the function "evalCoeff()". Within the implementation, the fading-out process is set to follow the S-curve shape depicted in Fig. 7 and corresponding to $\rho_{h}=0.5$, for which the perceived audio level is illustrated in Fig. 11. Anyhow, the fade shape can easily be controlled by means of the value received by parameter "rhoH" of function "evalCoeff()".

One observes that the "setInterval()" method of the "window" object, employed for discretization, calls the function "setFadingVol()" every $50 \mathrm{~ms}$ and passes the instant of fade-out initiation to that function. Moreover, function "setFadingVol()" is invoked until the value of rational fraction (1) comes to be less than or equal to 0 , when the audio content is paused and the "clearInterval()" method of the "window" object is used to clear the timer that has been set via the "setInterval()" method.

\section{Conclusion}

In the context of interactive multimedia, the major challenge is related to the development and realtime implementation of effective techniques for carrying out audio and video effects in an effort to optimize both the response time during effect progression and the delay between user intervention and the special effect initiation [1]-[4].

Traditionally, the fade audio effects are applied within different audio editors, via transcendental functions (logarithm, exponential, sine) to enforce the evolution of the audio level [10]-[14]. With a view to reactive computing, the present investigation advances a highly customizable fade-out audio effect by employing a convenient rational function to shape the fade profile. It is shown that the resulted fade effects can act not only similar to the fade-out effect of logarithmic or exponential profile but also as the fade effect of S-curve shape with its well-known attributes: flattened starting, flattened ending.

A plain HTML5/JavaScript application is made available in the paper with the purpose of checking the quality of the proposed shaping technique of being appropriate for interactive computing. The implementation appears to be straightforward, based on the extensively used properties and methods of the HTML DOM "audio" object. 


\section{References}

[1] V. COSTELlO: Multimedia Foundations. Core Concepts for Digital Design. New York, NY, USA, Routledge, 2016.

[2] M. SWEET: Writing Interactive Music for Video Games. A Composer's Guide. Upper Saddle River, NJ, USA, Addison-Wesley Professional, 2014.

[3] I. VIDAL, I. SOTO, et al.: Multimedia Networking Technologies, Protocols, and Architectures. Norwood, MA, USA, Artech House, 2019.

[4] K.C.C. YANG, et al.: Cases on Immersive Virtual Reality Techniques (Advances in Multimedia and Interactive Technologies). Hershey, PA, USA, IGI Global, 2019.

[5] I. DEVLIN: HTML5 Multimedia. Develop and Design. Berkeley, CA, USA, Peachpit Press, 2012.

[6] S. POWERS: HTML5 Media. Sebastopol, CA, USA, O’Reilly Media, 2011.

[7] N. WIRTH: A Plea for Lean Software. Computer, 28 (2), 64-68 (1995). doi: 10.1109/2.348001

[8] I. JACOBS, J. JAFFE, P. LE HEGARET: How the Open Web Platform Is Transforming Industry. IEEE Internet Computing, 16 (6), 82-86 (2012). doi:10.1109/MIC.2012.134

[9] L. LUPSA-TATARU: Implementing the Fade-in Audio Effect for Real-Time Computing. Applied Computer Science, 15 (2), 5-18 (2019). doi:10.23743/acs-2019-09

[10] W. JACKSON: Digital Audio Editing Fundamentals. Get Started with Digital Audio Development and Distribution. Berkeley, CA, USA, Apress Media, 2015. doi:10.1007/9781-4842-1648-4

[11] C. SCHRODER: The Book of Audacity. Record, Edit, Mix, and Master with the Free Audio Editor. San Francisco, CA, USA, No Starch Press, 2011.

[12] S. LANGFORD: Digital Audio Editing. Correcting and Enhancing Audio in Pro Tools, Logic Pro, Cubase, and Studio One. Burlington, MA, USA, Focal Press, 2014.

[13] A. U. CASE: Sound FX. Unlocking the Creative Potential of Recording Studio Effects. Burlington, MA, USA, Focal Press, 2007.

[14] J. D. REISS, A. McPHERSON: Audio Effects. Theory, Implementation and Application. Boca Raton, FL, USA, CRC Press, 2015.

[15] L. LUPSA-TATARU: Novel Technique of Customizing the Audio Fade-out Shape. Applied Computer Science, 14 (3), 5-14 (2018). doi:10.23743/acs-2018-17

[16] H. FASTL, E. ZWICKER: Psychoacoustics - Facts and Models (Springer Series in Information Sciences). Berlin, Germany, Springer-Verlag, 2007. doi:10.1007/978-3-540-68888-4

[17] W. M. HARTMANN: Signals, Sound, and Sensation (AIP Series in Modern Acoustics and Signal Processing). New York, NY, USA, Springer-Verlag, 1998.

[18] J. G. ROEDERER: The Physics and Psychophysics of Music. An Introduction. New York, NY, USA, Springer Science + Business Media, 2008. doi:10.1007/978-0-387-09474-8 